\title{
Switched Conditional PDF-Based Split VQ Using Gaussian Mixture Model
}

\author{
Saikat Chatterjee, Student Member, IEEE, and T. V. Sreenivas, Senior Member, IEEE
}

\begin{abstract}
In this letter, we develop switched conditional PDF-based split vector quantization (SCSVQ) method using the recently proposed conditional PDF-based split vector quantizer (CSVQ). The use of CSVQ allows us to alleviate the coding loss by exploiting the correlation between subvectors, in each switching region. Using the Gaussian mixture model (GMM)-based parametric framework, we also address the rate-distortion (R/D) performance optimality of the proposed SCSVQ method by allocating the bits optimally among the switching regions.

For the wideband speech line spectrum frequency (LSF) parameter quantization, it is shown that the optimum parametric SCSVQ method provides nearly 2 bits/vector advantage over the recently proposed nonparametric switched split vector quantization (SSVQ) method.
\end{abstract}

Index Terms-Gaussian mixture model (GMM), line spectrum frequency (LSF) coding, vector quantization.

\section{INTRODUCTION}

$\mathbf{M}$ OST of the speech coders use linear prediction (LP) analysis, and thus, more effective scheme of quantizing the LP coefficients (LPCs), equivalently line spectrum frequencies (LSFs), is in great demand. Vector quantization (VQ) of LSFs is the best way to reach lowest bitrate, but the prohibitive complexity of a full-search VQ limits its usage. Many different product code VQ methods [1], [6], [12], [15] have been reported for LSF coding, which reduce complexity with a moderate loss of quantization performance. One of the widely reported techniques is split VQ (SVQ) which was first proposed by Paliwal and Atal [2] for telephone-band speech and then further extended to wide-band speech [3], [5]. In SVQ, the LSF vector is split into subvectors, and then, the subvectors are quantized independently. This approach sacrifices the correlation between the subvectors and, thus, leads to a coding loss, referred to as "split loss" [10]. To recover the split loss, So and Paliwal [13], [14] have recently proposed switched split VQ (SSVQ) method, which provides better rate-distortion (R/D) performance than the traditional SVQ method. We also have proposed a parametric technique of conditional PDF-based split VQ (CSVQ) method to recover the split loss [15], [16]. The CSVQ was shown to perform better than SVQ, but poorer than SSVQ [15].

The SSVQ is a nonparametric product code VQ method, where the vector space is divided into non-overlapping Voronoi

Manuscript received May 15, 2007; revised August 31, 2007. The associate editor coordinating the review of this manuscript and approving it for publication was Prof. Alan McCree.

The authors are with the Department of Electrical Communication Engineering, Indian Institute of Science, Bangalore 560 012, India (e-mail: saikat@ece.iisc.ernet.in; tvsree@ece.iisc.ernet.in).

Digital Object Identifier 10.1109/LSP.2007.910284 regions $^{1}$ and a separate $S V Q$ is designed for each region. Thus, the SSVQ comprises of multiple SVQs. An input vector to be quantized is first classified to a Voronoi region and then the region-specific SVQ is used for quantization. It was shown [13], [14] that an increase in the number of Voronoi regions leads to better recovery of the split loss, but at the cost of a steep increase in complexity. For a practically reasonable number of Voronoi regions in SSVQ, we note that the region specific SVQs are susceptible to split loss. Also, another important point to note is that the SSVQ does not address the optimality of its R/D performance.

For a multivariate Gaussian source quantization, we have shown theoretically that the CSVQ performs better than SVQ [16]. Hence, we extend this formulation to a switched quantization technique in this letter, where we use separate CSVQ for each Voronoi region; the proposed method is referred to as switched CSVQ (SCSVQ). We also address the R/D performance optimality of the proposed SCSVQ method in a GMM-based parametric framework where the source PDF of each Voronoi region is modeled as a multivariate Gaussian. In this context, we would like to indicate that currently, there is a growing interest to develop parametric PDF-based quantization methods using the GMM [7], [8], [11]. For the parametric SCSVQ method, we derive the optimum bit allocation criteria among the Voronoi regions using the variable bitrate scheme [8]. Focusing on wideband speech LSF quantization, we show that the optimum parametric SCSVQ method provides nearly 2 bits/vector advantage over the nonparametric SSVQ method.

\section{PRELIMINARIES}

The SCSVQ consists of multiple CSVQs. Thus, we indicate the R/D performance optimality of CSVQ in this section, where as a practical CSVQ codec was developed in [15].

Let $\mathbf{X}$ be a $p$-dimensional vector which is multivariate Gaussian distributed as: $f_{\mathbf{X}}(\mathbf{x})=\mathbf{N}\left(\mu_{\mathbf{X}}, C_{\mathbf{X}}\right)$. We consider that the vector is split into $S$ number of subvectors, such that $\mathbf{X}=\left[\mathbf{X}_{1}^{T} \mathbf{X}_{2}^{T} \ldots \mathbf{X}_{S}^{T}\right]^{T}$. Let $p_{i}$ be the dimension of the $i$ th subvector $\mathbf{X}_{i}$, such that $\sum_{i=1}^{S} p_{i}=p$. To recover the split loss, we exploit the conditional PDF and sequentially quantize each subvector $\mathbf{X}_{i}, 1 \leq i \leq S$ [15], [16]; i.e., $\mathbf{X}_{i}$, is quantized utilizing the past information $\mathbf{Y}_{i}=\left[\mathbf{X}_{1}^{T} \mathbf{X}_{2}^{T} \ldots \mathbf{X}_{i-1}^{T}\right]^{T}$. We have shown that the optimum CSVQ method provides the R/D performance as (from [16, Theorem 2])

$$
\mathcal{D}_{C S V Q}=\left(2^{b}\right)^{-\frac{2}{p}} \mathcal{K}\left|C_{\mathbf{X}}\right|^{\frac{1}{p}}
$$

\footnotetext{
${ }^{1}$ These Voronoi regions are referred to as "switching regions" in [13] and [14] In this letter, Voronoi region and switching region are used interchangeably.
} 


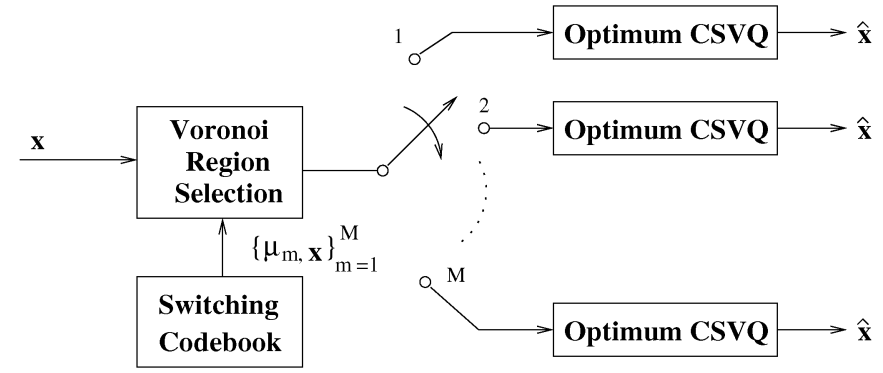

Fig. 1. GMM-based optimum switched conditional PDF-based split VQ (SCSVQ) method.

where $\mathcal{K}=\left[p\left[\prod_{i=1}^{S}\left(K_{p_{i}} / p_{i}\right)^{p_{i}}\right]^{1 / p}\right]$ and $K_{p_{i}}$ is a constant depending on the dimension $p_{i}$ as $K_{p_{i}}=$ $2\left[\left(p_{i} / 2\right) \Gamma\left(p_{i} / 2\right)\right]^{2 / p_{i}}\left(\left(p_{i}+2\right) / p_{i}\right)^{p_{i} / 2}[16] ; b$ is the allocated bits/vector to quantize the Gaussian source. To achieve the $\mathrm{R} / \mathrm{D}$ performance of (1), the optimum bit allocation among the subvectors is given by (from [16, Theorem 1])

$b_{i}=p_{i} \frac{b}{p}+\frac{p_{i}}{2} \log _{2}\left[\frac{\frac{1}{p_{i}} K_{p_{i}}\left|C_{\mathbf{X}_{i} \mathbf{X}_{i} \mid \mathbf{Y}_{i}}\right|^{\frac{1}{p_{i}}}}{\left[\prod_{j=1}^{S}\left(\frac{1}{p_{j}} K_{p_{j}}\right)^{p_{j}}\right]^{\frac{1}{p}}\left|C_{\mathbf{X}}\right|^{\frac{1}{p}}}\right], 1 \leq i \leq S$

where $b_{i}$ is the bits allocated to the $i$ th subvector $\left(\mathbf{X}_{i}\right)$ in CSVQ method, such that $\sum_{i=1}^{S} b_{i}=b ; C_{\mathbf{X}_{i} \mathbf{X}_{i} \mid \mathbf{Y}_{i}}$ is the conditional covariance matrix [16] of the $i$ th subvector $\left(\mathbf{X}_{i}\right)$.

\section{Switched CONDITIONAL PDF-BASEd SPLIT VQ}

In this section, we develop a new method of switched conditional PDF-based split VQ (SCSVQ) and address its R/D performance optimality using a parametric model of the source PDF. The basis of the SCSVQ method is to populate the vector space with $M$ number of CSVQs and switching to one of them for quantization, based on a nearest neighbor criterion. Fig. 1 shows a block diagram of the SCSVQ method. We address the issue of R/D performance optimality, using the Gaussian mixture model (GMM)-based framework. PDF of each switching region of SCSVQ is modeled as a multivariate Gaussian component of the GMM. Since the switching regions are nothing but Voronoi regions of the first stage quantizer, the mixture components of the GMM are assumed to be non-overlapping. Let $\mathbf{X}$ be the $p$-dimensional vector; the PDF of $\mathbf{X}$ is modeled using a Gaussian mixture (GM) density of $M$ components, given as

$$
f_{\mathbf{x}}(\mathbf{x}) \approx \sum_{m=1}^{M} \alpha_{m} \mathbf{N}\left(\mu_{m, \mathbf{x}}, C_{m, \mathbf{x}}\right)
$$

where $\alpha_{m}, \mu_{m, \mathbf{x}}$, and $C_{m, \mathbf{x}}$ are the prior probability, mean vector, and covariance matrix of the $m$ th Gaussian component $\mathbf{N}\left(\mu_{m, \mathbf{x}}, C_{m, \mathbf{x}}\right)$. The prior probabilities satisfy the stochastic constraint $\sum_{m=1}^{M} \alpha_{m}=1$. The approximate equality used in (3) is because of modeling a source PDF using the GMM.
While a direct R/D performance analysis of a GMM source is not easy to carry out [7], we consider a linearized approach [8] to the overall quantization distortion of the source, expressed as

$$
\mathcal{D}_{\mathbf{X}} \approx \mathcal{D}_{G M M}=\sum_{m=1}^{M} \alpha_{m} \mathcal{D}_{G, m}\left(b_{m}\right)
$$

where $\mathcal{D}_{G, m}\left(b_{m}\right)$ is the incurred distortion of the quantizer for the $m$ th component Gaussian of GMM and $b_{m}$ is the allocated $\mathrm{bits} / \mathrm{vector}$ to the quantizer. We need to solve for the optimum bit allocation, $b_{m}$, such that $\mathcal{D}_{G M M}$ is minimized. While there has been an attempt [7] to analyze the distortion incurred for a GM source using an expectation-maximization (EM) type of iterative algorithm, the above linearized approach provides a simple closed-form solution using the optimum inter-cluster bit allocation. Since the source PDF of each Voronoi region is modeled as a multivariate Gaussian with known parameters, we can use the high-resolution quantization distortion of CSVQ [16] for an overall distortion expression of the GM density and then optimize for $b_{m}$. The nature of approximation in (4) for $\mathcal{D}_{G M M}$ is that it is slightly overestimated because each bounded Voronoi region is treated as an unbounded Gaussian cluster, leading to a conservative estimate of the performance, while the actual distortion may be slightly lower.

The non-overlapping Voronoi regions (or switching regions) of SCSVQ method are formed using the LBG algorithm, and we compute the Gaussian parameters for each Voronoi region. The switching codebook consists of $\left\{\mu_{m, \mathbf{x}}\right\}_{m=1}^{M}$ as the codevectors.

\section{A. Inter-Cluster Bit Allocation}

The optimality of the SCSVQ method is addressed by achieving the best R/D performance through the optimum bit allocation among the component Gaussian clusters; this is referred to as inter-cluster bit allocation which can be carried out using the variable rate scheme of [8].

In a variable rate scheme, the average bits/vector $\left(b_{\text {avg }}\right)$ is fixed, but the actual bits/vector changes with input data. The first stage quantizer (switch) uses $\log _{2} M$ bits, followed by the second stage quantization (CSVQ) using $b_{m}$ bits for the $m$ th region. Thus, the coder becomes a variable rate coder because $b_{m}$ varies according to the changing signal characteristics. The variable bitrate constraint [8] is given by

$$
b_{a v g}=\sum_{m=1}^{M} \alpha_{m}\left(\log _{2} M+b_{m}\right)=\log _{2} M+\sum_{m=1}^{M} \alpha_{m} b_{m}
$$

The $b_{\text {avg }}$ is a design parameter, similar to a fixed rate coder. The optimum inter-cluster bit allocation is solved by minimizing the overall distortion, given in (4), to the constraint of average bits, shown in (5); i.e.,

$$
\begin{aligned}
\min _{b_{m}}\left\{\mathcal{D}_{G M M}=\sum_{m=1}^{M} \alpha_{m} \mathcal{D}_{G, m}\left(b_{m}\right)\right\}, \text { subject to } \\
b_{\text {avg }}=\log _{2} M+\sum_{m=1}^{M} \alpha_{m} b_{m} .
\end{aligned}
$$


Evaluation of $\mathcal{D}_{G M M}$ requires the best performance of $\mathcal{D}_{G, m}\left(b_{m}\right)$, which is given by the quantization distortion result of CSVQ [16] as [using (1)]

$$
\mathcal{D}_{G, m}\left(b_{m}\right)=\left(2^{b_{m}}\right)^{-\frac{2}{p}} \mathcal{K}_{m}\left|C_{m, \mathbf{X}}\right|^{\frac{1}{p}}
$$

where $\mathcal{K}_{m}=\left[p\left[\prod_{i=1}^{S_{m}}\left(K_{p_{m, i}} / p_{m, i}\right)^{p_{m, i}}\right]^{1 / p}\right]$. Here, $p_{m, i}$ is the dimension of the $i$ th split subvector for the $m$ th cluster-specific CSVQ method and $1 \leq i \leq S_{m}$. Note that, for the $m$ th region, the number of splits $\left(S_{m}\right)$ and the dimensions of split subvectors $\left(p_{m, i}\right)$ are design parameters which can be different for different Voronoi regions according to the choice of the designer. The optimization is solved using the Lagrange approach as given by the next theorem.

Theorem: The optimum inter-cluster bit allocation for SCSVQ method that minimizes the overall distortion $\mathcal{D}_{G M M}=$ $\sum_{m=1}^{M} \alpha_{m} \mathcal{D}_{G, m}\left(b_{m}\right)$, subject to the variable rate constraint of $b_{\text {avg }}=\log _{2} M+\sum_{m=1}^{M} \alpha_{m} b_{m}$, is given by

$$
b_{m}=\left(b_{\text {avg }}-\log _{2} M\right)+\frac{p}{2} \log _{2}\left[\begin{array}{r}
\left.\frac{\mathcal{K}_{m}\left|C_{m, \mathbf{X}}\right|^{\frac{1}{p}}}{\prod_{j=1}^{M}\left[\mathcal{K}_{j}\left|C_{j, \mathbf{X}}\right|^{\frac{1}{p}}\right]^{\alpha_{k}}}\right] \\
1 \leq m \leq M .
\end{array}\right.
$$

Proof:

$$
\begin{aligned}
\mathcal{L}= & \mathcal{D}_{G M M}+\lambda\left(\sum_{m=1}^{M} \alpha_{m} b_{m}+\log _{2} M-b_{\text {avg }}\right) \\
= & \sum_{m=1}^{M} \alpha_{m}\left(2^{b_{m}}\right)^{-\frac{2}{p}} \mathcal{K}_{m}\left|C_{m, \mathbf{X}}\right|^{\frac{1}{p}} \\
& +\lambda\left(\sum_{m=1}^{M} \alpha_{m} b_{m}+\log _{2} M-b_{\text {avg }}\right) .
\end{aligned}
$$

Using partial differentiation, $\partial \mathcal{L} / \partial b_{m}=0,1 \leq m \leq M$, we get

$$
b_{m}=-\frac{p}{2} \log _{2}\left[\frac{\lambda p}{2 \ln 2}\right]+\frac{p}{2} \log _{2}\left[\mathcal{K}_{m}\left|C_{m, \mathbf{X}}\right|^{\frac{1}{p}}\right] .
$$

Premultiplying both sides by $\alpha_{m}$, summing $\forall m$, and using $\sum_{m=1}^{M} \alpha_{m}=1$ and $b_{a v g}=\log _{2} M+\sum_{m=1}^{M} \alpha_{m} b_{m}$, we get

$$
\begin{aligned}
-\frac{p}{2} \log _{2}\left[\frac{\lambda p}{2 \ln 2}\right]=\left(b_{\text {avg }}\right. & \left.-\log _{2} M\right) \\
& -\frac{p}{2} \log _{2}\left[\prod_{m=1}^{M}\left[\mathcal{K}_{m}\left|C_{m, \mathbf{X}}\right|^{\frac{1}{p}}\right]^{\alpha_{m}}\right] .
\end{aligned}
$$

Substituting $-(p / 2) \log _{2}[\lambda p / 2 \ln 2]$ appropriately and using dummy variable $j$, we get the expression of (8).

\section{B. Intra-Cluster Bit Allocation}

The optimum bit allocation to the subvectors for CSVQ method [16] in each cluster (switching region) is given by [using (2)]

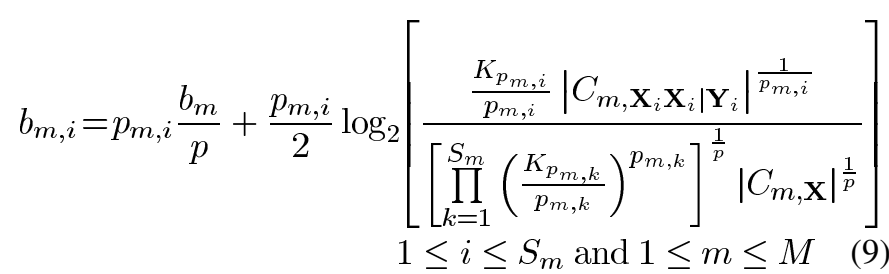

where $b_{m, i}$ is the number of bits allocated to the $i$ th sub-vector

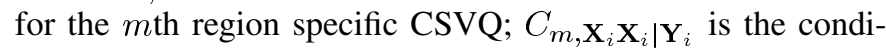
tional covariance matrix [16] of the $i$ th subvector for the $m$ th region.

\section{QUANTIZATION RESULtS}

To test the LSF quantization performance, we consider wideband speech LSFs and use the common measure of spectral distortion (SD) [2]. We also use another recently proposed measure of spectral distortion with interframe memory (SDM) [9]. A low average SD and rms SDM along with minimum number of high SD outliers are considered necessary for perceptually good spectrum quantization performance [2], [9], [13]. For better SD performance, the codebook is searched using the weighted square Euclidean distance (WSED) measure [2], [13], where the weights are the spectral sensitivity coefficients [4]. The speech data used in the experiments are from the TIMIT database. The specification of AMR-WB speech codec [17] is used to compute the 16th-order LPCs which are then converted to LSFs. We use $368815 \mathrm{LSF}$ vectors as training data and 87961 LSF vectors as test data (distinct from training data).

The parametric optimum SCSVQ and nonparametric SSVQ methods are implemented using $M=8$ Voronoi regions. For each Voronoi region, the 16 dimensional LSF vector is split into five parts of $(3,3,3,3,4)$ dimensional subvectors for both CSVQ and SVQ methods. The number of Voronoi regions and subvector dimensions are chosen according to [13]. The optimum parametric SCSVQ method is designed using variable bitrate scheme, resulting in fractional inter-cluster bit allocation [see (8)] and also fractional intra-cluster bit allocation [see (9)]. For the sake of practical implementation, we adjust the inter-cluster and intra-cluster fractional bits to integer quantities, satisfying the total bit budget. We also compare the performances of recently proposed CSVQ and traditional SVQ methods [15], using the same $(3,3,3,3,4)$ dimensional split.

The performance of parametric optimum SCSVQ method is shown in Table I. The nonparametric SSVQ is implemented according to [13], and the performance is shown in Table II. Tables III and IV, respectively, show the performances of CSVQ and SVQ methods. For CSVQ and SVQ, the bits are allocated to the subvectors uniformly such that they result in minimum complexity and the extra bits are allocated to those subvectors which results in least overall distortion [15]. For a visual comparison, the R/D performances of different methods are shown 
TABLE I

Performance of Optimum Switched Conditional PDF-Based SPLIT VQ (SCSVQ) METHOD

\begin{tabular}{ccccc}
\hline $\begin{array}{c}\text { Avg. bits/ } \\
\text { vector }\end{array}$ & $\begin{array}{c}\text { Avg. SD } \\
(\mathrm{dB})\end{array}$ & \multicolumn{2}{c}{ SD Outliers (in \%) } & RMS SDM \\
\cline { 3 - 4 } & $2-4 \mathrm{~dB}$ & $>4 \mathrm{~dB}$ & $(\mathrm{~dB})$ \\
\hline 42 & 1.05943 & 0.93564 & 0.00682 & 1.05656 \\
43 & 1.01977 & 0.75147 & 0.00682 & 1.01938 \\
44 & 0.98004 & 0.60822 & 0.00682 & 0.98294 \\
45 & 0.94188 & 0.45816 & 0.00455 & 0.94697 \\
46 & 0.90366 & 0.33992 & 0.00682 & 0.91110 \\
\hline
\end{tabular}

TABLE II

Performance of ReCEntLy Proposed NonParametric SWITCHED SPLIT VQ (SSVQ) METHOD

\begin{tabular}{ccccc}
\hline bits/vector & Avg. SD & \multicolumn{2}{c}{ SD Outliers (in \%) } & RMS SDM \\
\cline { 3 - 4 } & $(\mathrm{dB})$ & $2-4 \mathrm{~dB}$ & $>4 \mathrm{~dB}$ & $(\mathrm{~dB})$ \\
\hline 42 & 1.12381 & 1.28921 & 0.00114 & 1.11868 \\
43 & 1.07195 & 0.85038 & 0.00114 & 1.07017 \\
44 & 1.03659 & 0.72077 & 0.00114 & 1.04079 \\
45 & 1.00399 & 0.59117 & 0.00000 & 1.00933 \\
46 & 0.96795 & 0.46498 & 0.00000 & 0.97411 \\
\hline
\end{tabular}

TABLE III

Performance of Recently Proposed Conditional PDF-Based SPLIT VQ (CSVQ) METHOD

\begin{tabular}{ccccc}
\hline bits/vector & $\begin{array}{c}\text { Avg. SD } \\
\end{array}$ & \multicolumn{2}{c}{ SD Outliers (in \%) } & RMS SDM \\
\cline { 3 - 4 } & $(\mathrm{dB})$ & $2-4 \mathrm{~dB}$ & $>4 \mathrm{~dB}$ & $(\mathrm{~dB})$ \\
\hline 42 & 1.15190 & 1.99975 & 0.00000 & 1.13574 \\
43 & 1.11509 & 1.57001 & 0.00000 & 1.10072 \\
44 & 1.07933 & 1.38470 & 0.00000 & 1.07142 \\
45 & 1.01577 & 0.73328 & 0.00000 & 1.01495 \\
46 & 0.97706 & 0.58208 & 0.00000 & 0.97803 \\
\hline
\end{tabular}

TABLE IV

Performance of Traditional SPlit VQ (SVQ) Method

\begin{tabular}{ccccc}
\hline bits/vector & $\begin{array}{c}\text { Avg. SD } \\
\text { (dB) }\end{array}$ & \multicolumn{2}{c}{ SD Outliers (in \%) } & RMS SDM \\
\cline { 3 - 4 } & $2-4 \mathrm{~dB}$ & $>4 \mathrm{~dB}$ & $(\mathrm{~dB})$ \\
\hline 42 & 1.25809 & 2.63753 & 0.00000 & 1.22922 \\
43 & 1.21474 & 2.02817 & 0.00000 & 1.18928 \\
44 & 1.18267 & 1.83263 & 0.00000 & 1.16339 \\
45 & 1.11602 & 0.97430 & 0.00000 & 1.10505 \\
46 & 1.07404 & 0.74465 & 0.00000 & 1.06600 \\
\hline
\end{tabular}

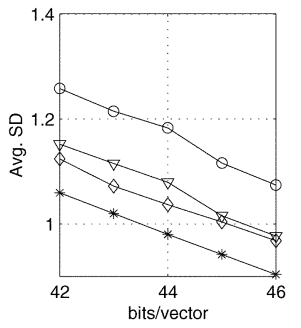

(a)

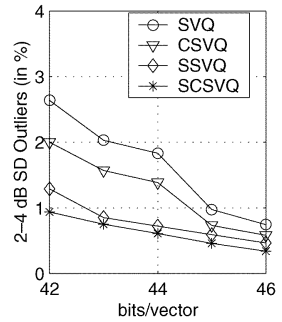

(b)

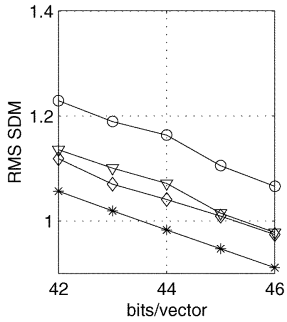

(c)
Fig. 2. Rate-distortion performance comparison between split VQ (SVQ), conditional PDF-based split VQ (CSVQ), switched split VQ (SSVQ), and switched conditional PDF-based split VQ (SCSVQ) methods. (a) Average SD (in dB) performance. (b) 2-4 dB outlier (in \%) performance. (c) RMS SDM (in dB) performance.

in Fig. 2. It can be seen that the SCSVQ provides the best performance in terms of all the three performance measures. As expected, the next best is SSVQ, which outperforms the traditional SVQ. We note that the CSVQ performs poorer than SSVQ. In terms of average SD measure, the nonparametric SSVQ method provides 2-3 bits/vector advantage over SVQ. The GMM-based optimum SCSVQ method provides a further 2 bits/vector advantage over SSVQ method. These advantages are valid for the rms SDM measure also.

\section{CONCLUSION}

We propose an optimum switched conditional PDF-based split VQ (SCSVQ) method using the Gaussian mixture model-based framework. The rate-distortion performance optimality of SCSVQ method is addressed using a linearized approximation of the total average distortion, resulting in optimum inter-cluster and intra-cluster bit allocation. For wideband speech LSF quantization, we show that the new parametric optimum SCSVQ method provides nearly 2 bits/vector advantage over the recently proposed nonparametric switched split VQ (SSVQ) method and 4 bits/vector advantage over the traditional SVQ method.

\section{REFERENCES}

[1] R. Laroia, N. Phamdo, and N. Farvardin, "Robust and efficient quantization of speech LSP parameters using structured vector quantizers," in Proc. ICASSP, 1991, pp. 641-644.

[2] K. K. Paliwal and B. S. Atal, "Efficient vector quantization of LPC parameters at 24 bits/frame," IEEE Trans. Acoust., Speech, Signal Process., vol. 1, pp. 3-14, Jan. 1993.

[3] R. Lefebvre, R. Salami, C. Laflamme, and J. P. Adoul, "High quality coding of wide-band audio signals using transform coded excitation (TCX)," in Proc. ICASSP, 1994, pp. 193-196.

[4] W. R. Gardner and B. D. Rao, "Theoretical analysis of the high-rate vector quantization of LPC parameters," IEEE Trans. Speech Audio Process., vol. 3, no. 5, pp. 367-381, Sep. 1995.

[5] J. H. Chen and D. Wang, "Transform predictive coding of wide-band speech signals," in Proc. ICASSP, 1996, pp. 275-278.

[6] D. Chang, S. Ann, and C. W. Lee, "A classified vector quantization of LSF parameters," Signal Process., vol. 59, pp. 267-273, Jun. 1997.

[7] P. Hedelin and J. Skoglund, "Vector quantization based on Gaussian mixture models," IEEE Trans. Speech Audio Process., vol. 8, no. 4, pp. 385-401, Jul. 2000.

[8] A. D. Subramaniam and B. D. Rao, "PDF optimized parametric vector quantization of speech line spectral frequencies," IEEE Trans. Speech Audio Process., vol. 11, no. 2, pp. 130-142, Mar. 2003.

[9] F. Norden and T. Eriksson, "Time evolution in LPC spectrum coding," IEEE Trans. Speech Audio Process., vol. 12, no. 3, pp. 290-301, May 2004.

[10] F. Norden and T. Eriksson, "On split quantization of LSF parameters," in Proc. ICASSP, May 2004, vol. 1, pp. I-157-I-160.

[11] A. D. Subramaniam, W. R. Gardner, and B. D. Rao, "Low-complexity source coding using Gaussian mixture models, lattice vector quantization, and recursive coding with application to speech spectrum quantization," IEEE Trans. Audio, Speech, Lang. Process., vol. 14, no. 2, pp. 524-532, Mar. 2006.

[12] S. Chatterjee and T. V. Sreenivas, "Two stage transform vector quantization of LSFs for wideband speech coding," in Proc. Int. Conf. Spoken Language Processing (INTERSPEECH), Pittsburgh, PA, Sep. 2006, pp. 233-236.

[13] S. So and K. K. Paliwal, "A comparative study of LPC parameter representations and quantisation schemes for wide-band speech coding," Digit. Signal Process., vol. 17, no. 1, pp. 114-137, Jan. 2007.

[14] S. So and K. K. Paliwal, "Efficient product code vector quantisation using the switched split vector quantiser," Digit. Signal Process., vol. 17, no. 1, pp. 138-171, Jan. 2007.

[15] S. Chatterjee and T. V. Sreenivas, "Conditional PDF-based split vector quantization of wideband LSF parameters," IEEE Signal Process. Lett., vol. 14, no. 9, pp. 641-644, Sep. 2007.

[16] S. Chatterjee and T. V. Sreenivas, "Analysis of conditional PDF based split VQ,” IEEE Signal Process. Lett., accepted for publication.

[17] AMR Wide-Band Speech Codec, Transcoding Functions (Release 5): 3GPP TS 26.190 V 5.1.0. 